\title{
Desenvolvimento do amendoim-bravo (Pterogyne nitens Tul.) sob lâminas de água e composições de substratos
}

\author{
Amanda Rossetto Pacheco ${ }^{\mathrm{a}}$, Miguel Stancare Neto ${ }^{\mathrm{a}} \odot$, Gustavo Henrique Gravatim Costa ${ }^{\mathrm{b}}$, \\ Marcelo da Fossa Paz ${ }^{\mathfrak{c}}$, Marcos Vinicius Bohrer Monteiro Siqueira ${ }^{b *} \mathbb{0}$
}

\footnotetext{
a Universidade do Sagrado Coração, Brasil

${ }^{\mathrm{b}}$ Universidade do Estado de Minas Gerais, Unidade Frutal, Brasil

${ }^{\text {c } U n i v e r s i d a d e ~ F e d e r a l ~ d a ~ G r a n d e ~ D o u r a d o s, ~ B r a s i l ~}$

*Autor correspondente (mvbsiqueira@gmail.com)
}

\section{N F O}

\section{Keyworks}

biosolid

composted sewage sludge

plant nutrition

seedling production

\section{Palavras-chaves \\ biossólido \\ lodo de esgoto \\ compostado \\ nutrição mineral de \\ plantas \\ produção de muda}

\section{A B S T R A C T}

Development of amendoim-bravo (Pterogyne nitens Tul.) in water slides and substrate compositions. The wild peanut (Pterogyne nitens Tul.) is considered a rustic species and adaptable to several regions of the national territory, and indicated for silviculture, plantations in degraded areas and replacement of riparian forests. The objective of this work was to evaluate the development of wild peanut seedlings under different concentrations of composted sewage sludge (LEC), commercial substrate (SC) and water depths. The experimental design consisted of 240 seedlings, divided into five different treatments: S1 (100\% LEC), S2 (75\% LEC + 25\% SC), S3 (50\% LEC + 50\% SC), S4 (25\% LEC + 75\% SC) and S5 (100\% SC). The experiment had three irrigation depths, four times a day: L1 (6 minutes), L2 (9 minutes) and L3 (12 minutes). The following characteristics were evaluated: height of the aerial part, diameter of the collection, number of leaves, weight of the dry mass and the Dickson Quality Index. The data were subjected to analysis of variance and the measures compared using the Tukey test. Treatments with $75 \%$ to $100 \%$ of SCF showed superior results in the evaluated parameters in relation to SC. Regarding the water regime, there was no significant influence of the water depths on the morphological characteristics of the seedlings.

\section{R E S U M O}

O amendoim-bravo (Pterogyne nitens Tul.) é considerado uma espécie rústica e adaptável a várias regiões do território nacional, e indicada para silvicultura, plantios em áreas degradas e reposição de matas ciliares. O objetivo deste trabalho foi avaliar o desenvolvimento das mudas de amendoim-bravo sob concentrações de lodo de esgoto compostado (LEC), substrato comercial (SC) e lâminas de água. O delineamento experimental foi composto por 240 mudas, divididas em cinco tratamentos diferentes: S1 (100\% LEC), S2 (75\% LEC + 25\% SC), S3 (50\% LEC + 50\% SC), S4 (25\% LEC + 75\% SC) e S5 ( $100 \%$ SC). O experimento contou com três lâminas de irrigação, quatro vezes ao dia: L1 (6 minutos), L2 (9 minutos) e L3 (12 minutos). Foram avaliadas as seguintes características: altura da parte aérea, diâmetro do coleto, número de folhas, peso da massa seca e o Índice de Qualidade de Dickson. Os dados foram submetidos a análise de variância e as medidas comparadas pelo teste de Tukey. Tratamentos com $75 \%$ a $100 \%$ de LEC apresentaram resultados superiores nos parâmetros avaliados em relação ao SC. Para o regime hídrico, não houve influência significativa das lâminas de água sobre as características morfológicas das mudas. 


\section{INTRODUÇÃO}

A espécie Pterogyne nitens Tul. é conhecida popularmente como amendoim-bravo. Pertence à família Fabaceae, ocorre naturalmente entre as latitudes $06^{\circ} \mathrm{S}$ a $28^{\circ}$, e é uma espécie secundária inicial, comportando-se como pioneira; ocorre em locais arenosos e degradados e em solos de baixa fertilidade natural, é recomendada para plantios em reposição de matas ciliares em locais com inundações periódicas de rápida duração e recomposição e restauração de áreas degradadas (Lorenzi, 2002; Biruel et al., 2007; Santos et al., 2018).

Com a constante exploração das florestas nativas pela expansão do agronegócio, tem-se observado uma redução da cobertura florestal, gerando uma grande demanda por mudas nativas para a recuperação de áreas degradadas e reflorestamento (Leles et al., 2006; Caldeira et al., 2013; Siqueira et al., 2017).

O desenvolvimento de novas técnicas para a produção de mudas florestais é de extrema importância econômica e ambiental, bem como a inovação e busca por redução de gastos com rápidos ganhos (Weiss et al., 2020). A utilização de resíduos provenientes das estações de tratamento de esgoto traz resultados relevantes, principalmente como substrato para espécies arbóreas destinadas à restauração florestal (Rebouças et al., 2010; Siqueira et al., 2018).

Quando tratado, o lodo de esgoto compostado (LEC), pode trazer benefícios ao meio ambiente, por intermédio da sua reutilização como substrato, melhorando a fertilidade de solos degradados (Bezerra et al., 2006; Nogueira et al., 2009; Cieslik et al., 2015). Esse material apresenta alto teor de matéria orgânica, como nitrogênio e fósforo, além de micronutrientes e elevada retenção de água (Vega et al., 2004; Santos et al. 2014; Nobrega et al., 2017).

Uma das etapas importantes na produção de mudas de qualidade é a irrigação, sendo um fator que influencia diretamente no crescimento e desenvolvimento de qualquer espécie. O déficit hídrico, pode ocasionar deficiência na absorção de nutrientes; já o excesso pode favorecer a lixiviação dos nutrientes, proporcionando um microclima favorável para doenças (Morais et al., 2012).

Nesse sentido, o LEC nos últimos anos tem recebido significativa atenção de viveiros quanto ao seu uso na formulação de substratos para mudas de espécies florestais (Pedrosa et al., 2017). O LEC foi utilizado como substrato para produção de diversas mudas nativas como o dedaleiro (Santana et al., 2019), embaúba (Morgado et al., 2020), a quaresmeira (Lobo et al., 2018), o monjoleiro (Lanzeti et al., 2021), entre outras.
Com este trabalho objetivou-se analisar o desenvolvimento do amendoim-bravo (Pterogyne nitens Tul.) em diferentes composições de LEC e lâminas de irrigação.

\section{MATERIAL E MÉTODOS}

\section{Local de estudo}

O experimento foi conduzido no viveiro Associação de Recuperação Florestal e Ecológica (ACIFLORA), localizado em Bauru, SP. Segundo a classificação de Koppen, o clima da região é tropical de savana, tipo Aw, com uma média de precipitação anual de $1.331 \mathrm{~mm}$ e de temperatura com máxima de $32^{\circ} \mathrm{C}$ e mínima de $20^{\circ} \mathrm{C}$ (Alvares et al., 2013).

\section{Montagem do experimento}

As sementes da espécie foram fornecidas pelo Instituto Florestal de São Paulo. A semeadura foi realizada a partir da distribuição em fileiras dentro de um canteiro construído com tábuas de madeira, com areia fina localizado no chão e com dimensão de $1 \mathrm{~m} \times 20 \mathrm{~m}$. Finalizada a semeadura, cobriu-se com vermiculita e revestiu-se com um telado de sobreamento (50\%). Após um mês da semeadura, as mudas foram repicadas e transplantadas para 240 tubetes de $120 \mathrm{~cm}^{3}$ cada, sendo então mantidas por um período de 45 dias em casa de vegetação.

$\mathrm{O}$ delineamento estatístico utilizado foi o de blocos casualizados, composto por 240 mudas, submetidas a 15 tratamentos compostos pela combinação de cinco substratos e três lâminas de irrigação, havendo quatro repetições por tratamento conforme descrito por Lobo et al. (2018), Santana et al. (2019), Morgado et al. (2020), Olher et al. (2020), Oliveira et al. (2020) e Lanzeti et al. (2021).

\section{Substratos}

Os substratos utilizados foram o Carolina Soil Florestal ${ }^{\circledR}$, identificado como substrato comercial (SC) segundo o fabricante, o composto é formado por turfa de Sphagno, vermiculita expandida, casca de arroz carbonizada, e traços de NPK, calcário dolomítico e gesso agrícola, e o composto orgânico Terafertil, identificado como substrato de lodo de esgoto compostado, LEC, e fornecido pela empresa Tera Ambiental do município de Jundiaí-SP. A partir dos dois substratos foram formuladas as seguintes combinações de substratos: S1: 100\% LEC; S2: 75\% LEC + 25\% SC; S3: $50 \%$ LEC + $50 \%$ SC; S4: $25 \%$ LEC + 75\% SC e S5: Controle $100 \%$ SC.

\section{Irrigação}

A irrigação foi realizada por microaspersores tipo Fog, com bicos do modelo mist, com vazão de $120 \mathrm{~L} \mathrm{~h}^{-1} \mathrm{e}$ separados em três diferentes lâminas: L1 
com 6 minutos de irrigação $(10 \mathrm{~mm})$; L2 com 9 minutos de irrigação (16 mm) e L3 com 12 minutos de irrigação $(21 \mathrm{~mm})$, sendo cada lâmina submetida a quatro irrigações ao dia.

\section{Avaliações biomédicas e análise estatística}

Durante o período de janeiro a outubro de 2018 de experimento foram mensurados, mensalmente, a altura da parte aérea (determinada a partir do nível de substrato até a ponta da última folha), diâmetro do coleto (determinado por paquímetro e calculado ao nível do substrato), número de folhas e peso seco da parte aérea e da raiz.

Após a medição final, as mudas foram cortadas à altura do colo, separando-se a parte aérea da raiz. Pesou-se o material fresco em balança digital (Shimadzu, modelo AY220) e posteriormente, o material foi levado a uma estufa (Fanem, modelo $315 \mathrm{SE})$ por um período de $72 \mathrm{~h} \mathrm{em}$ uma temperatura de $60{ }^{\circ} \mathrm{C}$ para a sua secagem e determinação da matéria seca.

A qualidade da muda foi avaliada por meio do Índice de Qualidade de Dickson (Dickson et al., 1960), conforme a Equação 1:

$$
I Q D=\frac{P M S T}{\frac{H}{D C}+\frac{P M S P A}{P M S R}}
$$

Onde:

PMST: Produção da Massa Seca Total (g);

\author{
H: Altura (cm); \\ DC: Diâmetro do Colo (mm) \\ PMSPA: Produção da Massa Seca Aérea (g); \\ PMSPR: Produção da Massa Seca Radicular \\ (g).
}

A taxa de crescimento foi calculada a partir do coeficiente angular $(\operatorname{tg} \alpha)$ da linha de regressão linear obtida pela média dos valores de crescimento.

Os dados foram submetidos a análise de variância e as medidas comparadas pelo teste de Tukey à nível 5\%. Os dados cinéticos foram submetidos a análise de regressão.

\section{RESULTADOS E DISCUSSÃO}

\section{Altura das mudas}

Os substratos formulados com maiores concentrações de LEC obtiveram resultados superiores em altura. A diferenciação dos tratamentos se evidenciou a partir do nono mês, onde constatou-se que mudas em S1 (100\% LEC), S2 (75\% LEC + 25\% SC) e S3 (50\% LEC + 50 SC) obtiveram melhores resultados quando comparados com os tratamentos S4 (25\% LEC + 75\% SC) e S5 $(100 \%$ SC). Verificou-se que as maiores médias obtidas foram em S2 (75\% LEC + 25\% SC) e S3 $(50 \%$ LEC $+50 \%$ SC) (Tabela 01).

$\underline{\text { Tabela } 1 \text { - Influência dos substratos no crescimento (altura em mm) de amendoim-bravo (Pterogyne nitens). }}$

\begin{tabular}{cccccc}
\hline \multirow{2}{*}{$\begin{array}{c}\text { Tempo } \\
\text { (dias) }\end{array}$} & \multicolumn{5}{c}{$\begin{array}{c}\text { Tratamento } \\
(\mathrm{mm})\end{array}$} \\
\cline { 2 - 6 } & $\mathrm{S} 1$ & $\mathrm{~S} 2$ & $\mathrm{~S} 3$ & $\mathrm{~S} 4$ & $\mathrm{~S} 5$ \\
\hline 30 & $5,45 \pm 0,49$ & $5,61 \pm 0,50$ & $5,83 \pm 0,57$ & $5,57 \pm 0,40$ & $5,50 \pm 0,48$ \\
60 & $11,25 \pm 0,89$ & $12,10 \pm 0,40$ & $12,44 \pm 1,33$ & $11,86 \pm 0,80$ & $11,24 \pm 0,95$ \\
90 & $18,15 \pm 1,38$ & $19,86 \pm 0,22$ & $20,21 \pm 1,90$ & $19,10 \pm 0,89$ & $17,92 \pm 1,32$ \\
120 & $25,62 \pm 1,86$ & $28,10 \pm 0,02$ & $28,12 \pm 2,31$ & $26,70 \pm 1,15$ & $25,05 \pm 1,55$ \\
150 & $33,85 \pm 1,96$ & $36,26 \pm 0,18$ & $36,56 \pm 2,44$ & $34,19 \pm 1,13$ & $32,166 \pm 1,87$ \\
180 & $41,37 \pm 2,56$ & $44,05 \pm 0,11$ & $44,32 \pm 2,69$ & $41,56 \pm 1,39$ & $39,05 \pm 2,22$ \\
210 & $49,27 \pm 3,27$ & $51,98 \pm 0,27$ & $52,14 \pm 3,03$ & $49,02 \pm 1,65$ & $46,01 \pm 2,53$ \\
240 & $58,09 \pm 3,32$ & $60,09 \pm 0,09$ & $60,33 \pm 3,18$ & $56,55 \pm 1,59$ & $53,00 \pm 2,91$ \\
270 & $67,67 \pm 4,29$ & $69,65 \pm 0,38$ & $69,90 \pm 3,79$ & $64,94 \pm 2,02$ & $60,49 \pm 3,55$ \\
300 & $77,27 \pm 4,77$ & $79,23 \pm 0,31$ & $79,26 \pm 4,15$ & $73,16 \pm 2,51$ & $67,91 \pm 4,31$ \\
\hline Taxa de Cresc. & 0,027 & 0,027 & 0,027 & 0,026 & 0,023 \\
$(\mathrm{~mm} /$ dia)* & 0,9959 & 0,9984 & 0,9985 & 0,9992 & 0,9994 \\
$\mathrm{R}^{2}$ & & & & & \\
\hline
\end{tabular}

*Obtida pelo coeficiente angular da curva de crescimento. S1: 100\% LEC; S2: 75\% LEC + 25\% SC; S3: 50\% LEC + 50\% SC; S4: $25 \%$ LEC + 75\% SC e S5: Controle $100 \%$ SC.

A altura da parte aérea é utilizada para estimar o padrão de qualidade de mudas de espécies florestais em viveiros, no entanto, para espécies nativas, os parâmetros adequados de altura, ainda não estão definidos para as diversas espécies florestais
(Oliveira et al., 2014).

Os resultados de altura neste estudo indicam que o uso do LEC traz vantagens em relação ao substrato comercial. Resultados semelhantes foram encontrados por Paiva et al. (2009), nos quais a 
utilização de LEC se equiparou com a utilização de fertilizantes minerais sob o desenvolvimento das espécies de aroeira-pimenteira (Schinus terebinthifolius Raddi), cabreúva-vermelha (Myroxylon peruiferum L.f.), pau-de-viola (Citharexylum myrianthum Cham.) e unha-de-vaca (Bauhinia forficata Link.). No entanto, Scheer et al. (2012) avaliaram que no desenvolvimento das mudas de angico-gurucaia (Parapiptadenia rigida Benth.) o LEC puro proporcionou uma nutrição às plantas, superior ao SC, o que não aconteceu com a espécie estudada já que o $\mathrm{S} 1(100 \%$ LEC $)$ apresentou média de crescimento menor em S2 e S3.

Os benefícios do uso de LEC foram também observados por Caldeira et al. (2014), com mudas de Eucalyptus grandis (W. Hill), misturando o LEC com resíduos vegetais (fibra de coco e palha de café in natura), onde os maiores crescimentos foram observados nos tratamentos S1 (100\% LEC), e S2
(80\% LEC + 20\% Fibra de Coco).

Trigueiro e Guerrini (2014) mostraram que combinações de SC contendo LEC apresentaram resultados superiores para espécies florestais de rápido crescimento, o que se observa nos resultados desse trabalho. $\mathrm{O}$ incremento na altura está relacionada aos acréscimos de matéria orgânica, substratos ricos em matéria orgânica apresentam um melhor desenvolvimento das mudas, gerando uma boa formação do sistema radicular (Faustino et al., 2005).

Em relação às lâminas de água, não ocorreram variações estatísticas significantes entre L1, L2 e L3 ao final de 300 dias de medições das mudas. A quantidade de água e o tempo usado em cada lâmina não interferiram na eficiência do substrato, em relação à altura da parte aérea, sendo suficientes 4 irrigações diárias de 6 minutos $(10 \mathrm{~mm})$ cada (Figura 01).

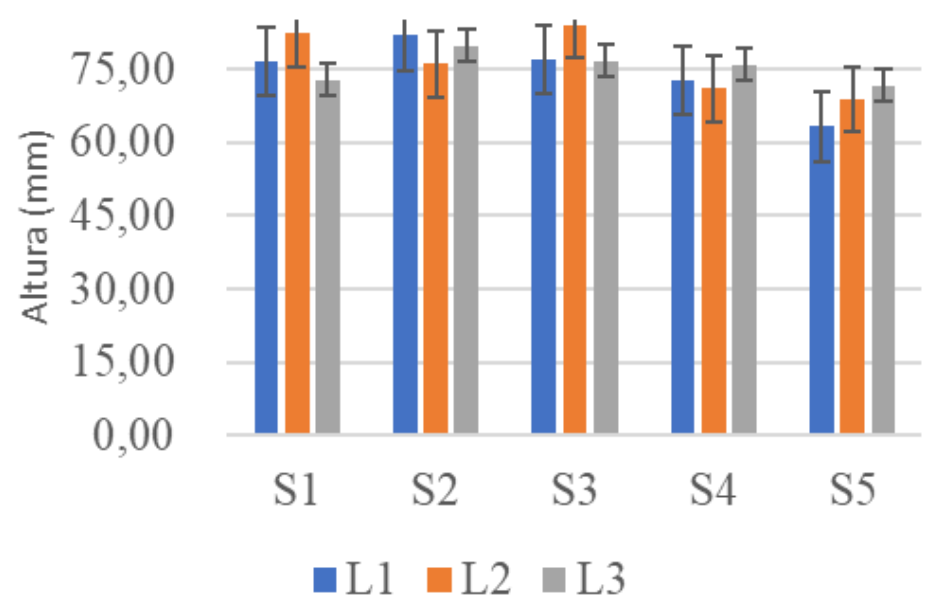

S1: 100\% LEC; S2: 75\% LEC + 25\% SC; S3: 50\% LEC + 50\% SC; S4: 25\% LEC + 75\% SC e S5: Controle 100\% SC. L1: 6 minutos de irrigação (10 mm); L2: 9 minutos de irrigação $(16 \mathrm{~mm})$, L3: 12 minutos de irrigação $(21 \mathrm{~mm})$.

Figura 1 - Influência das lâminas de água em relação à altura das mudas (mm) de amendoim-bravo (Pterogyne nitens Tul.).

Em um estudo com mudas de aroeira-pimenteira (Schinus terebinthifolius Raddi), Trigueiro e Guerrini (2014) observaram que, em relação a característica física dos substratos, à medida que se aumenta a proporção de LEC na mistura, há um aumento da sua densidade, ocorrendo uma redução da macroporosidade e aumento da microporosidade do substrato, proporcionando uma maior capacidade de retenção de água. Esta afirmação pode explicar a tendência de comportamento observada na Figura 01, ainda que não haja significância estatística.

\section{Diâmetro do Coleto}

Durante o experimento, observou-se que até o quarto mês as mudas de $P$. nitens (Tul.) apresentaram um diâmetro do coleto de $1 \mathrm{~mm}$. Entre o quinto e o sexto mês de avaliação o diâmetro do coleto apresentou uma média de $2 \mathrm{~mm}$ em todos os tratamentos, e após o nono mês o diâmetro do coleto foi de $3 \mathrm{~mm}$. Observou-se que não houve significância entre os resultados obtidos para este parâmetro, tanto para as lâminas de água como na composição de substrato em discordância com o trabalho de Scheer et al. (2012), no qual, o diâmetro do coleto apresentou aumento significativo com o uso de substratos à base de LEC, sem adição de fertilizantes, para as mudas de angico-branco (Anadenanthera colubrina Vell.).

$\mathrm{O}$ diâmetro do coleto está entre um dos 
principais fatores que irão determinar a qualidade das mudas. Junto a este parâmetro, são também incluídos a altura e o peso da parte aérea e subterrânea, sendo que plantas com maior diâmetro do coleto possibilitam melhor equilíbrio do crescimento da parte aérea (Gomes e Paiva, 2004; Rossa et al., 2015). Essa característica morfológica, em geral, é a mais observada para avaliar a capacidade de sobrevivência de uma muda em campo e é considerado um dos parâmetros que melhor refletem a qualidade de mudas florestais (Melo et al., 2018).

Bomfim et al. (2009), observaram médias do diâmetro do coleto de $3,18 \mathrm{~mm}$ para mudas produzidas em tubetes de $288 \mathrm{~cm}^{3}$ e $1,95 \mathrm{~mm}$ para mudas em tubetes de $50 \mathrm{~cm}^{3}$. No presente trabalho, o diâmetro de $3 \mathrm{~mm}$, ocorreu a partir do nono mês de desenvolvimento em tubetes de $120 \mathrm{~cm}^{3}$. Observou-se que o tamanho do tubete influenciou diretamente na espessura do coleto, atribuído à quantidade e/ou qualidade de substrato fornecido. A escolha do tamanho adequado de tubetes é fundamental para o desenvolvimento radicular visto que, tubetes menores apresentam maior restrição radicular, o que acarreta menor desenvolvimento da planta (Santos et al., 2000).

\section{Número de folhas}

$\mathrm{O}$ número de folhas apresentou um destaque quando correlacionada com a altura das mudas em relação ao LEC, onde S1 e S2 se diferenciaram significativamente na quantidade de folhas contabilizadas após 300 dias de desenvolvimento. Nesse parâmetro morfológico, S1 apresentou maiores médias no número de folhas em relação aos outros substratos utilizados, sendo superior a 40 folhas; já S2 possuiu uma média de 40 folhas e S3, S4 e S5 obtiveram valores inferiores (Figura 02).

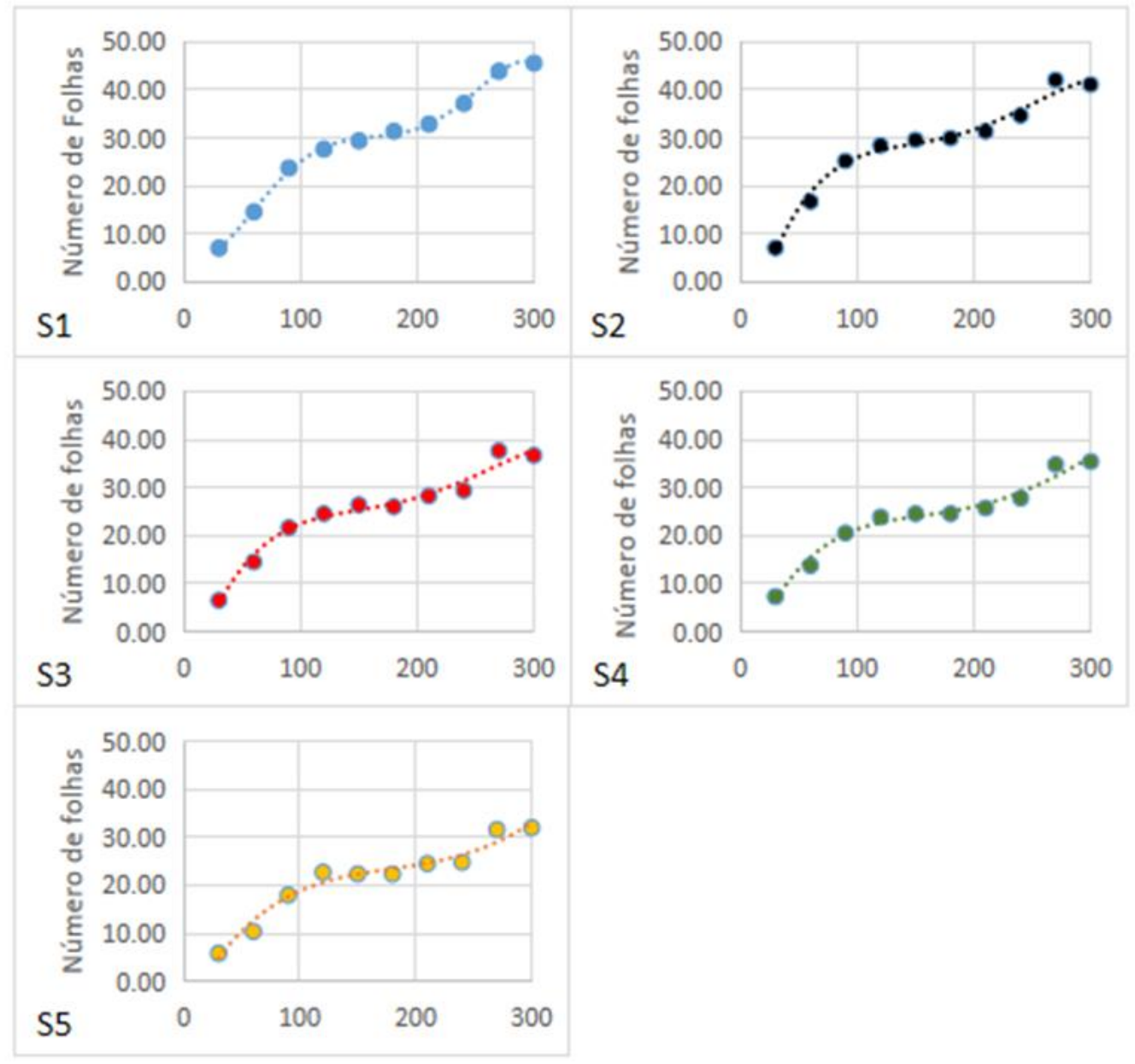

Eixo X representa o tempo (dias). Eixo Y representa o número de folhas. S1, curva em azul; S2, curva em preto; S3, curva em vermelho; S4, curva em verde; S5, curva em laranja.

Figura 2 - Influência dos substratos em relação ao número de folhas para as mudas de amendoim-bravo (Pterogyne nitens Tul.).

As curvas de aumento do número de folha se ajustaram perfeitamente ao modelo de regressão 
polinomial de $4^{\circ}$ grau com confiança de $99,2 \%$ para $\mathrm{S} 1,98,8 \%$ para $\mathrm{S} 2,98,9 \%$ para $\mathrm{S} 3,97,8 \%$ para $\mathrm{S} 4$ e $98,2 \%$ para S5, isso significa que o fenômeno observado nas curvas (Figura 02), mostram o fenômeno real, onde, para todos os substratos houve aumento no número de folhas nos primeiros 100 dias, uma parada entre os dias 100 e 200, retomando o aumento e estabilizando novamente em torno do dia 300 mostrando ciclos de 100 dias entre as fases de aumento e estabilidade.

Os resultados quanto ao número de folhas estão em concordância com Morgado et al. (2020) que trabalharam com Cecropia pachystachya (Trécul.) que encontraram maior número de folhas nas combinações de substratos do que nos substratos puros.

Para esse parâmetro (número de folhas) a redução de macroporosidade com subsequente aumento na retenção de água observada por Trigueiro e Guerrini (2014) explica de forma mais evidente o que foi observado na figra 2 onde os substratos com maiores quantidades de LEC apresentaram maior número de folhas.

Em relação ao regime hídrico, não houve diferenças significativas, contudo, L1 apresentou maiores médias em relação ao número de folhas (Figura 03) demonstrando, novamente, que a quantidade de água disponibilizada com L1 (10 $\mathrm{mm}$ ) já é suficiente para o desenvolvimento das mudas.

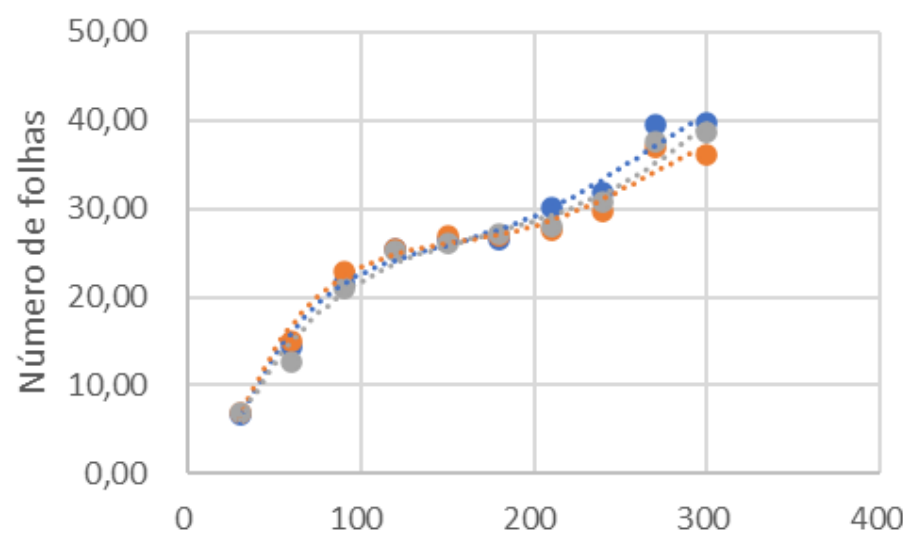

Lâmina 1 Lâmina 2 Lâmina 3

Legenda: Eixo X representa o tempo (dias). Eixo Y representa o número de folhas. L1, curva em Azul; L2, curva em laranja e L3, curva em cinza. L1: 6 minutos de irrigação $(10 \mathrm{~mm})$; L2: 9 minutos de irrigação $(16 \mathrm{~mm})$, L3: 12 minutos de irrigação $(21 \mathrm{~mm})$.

Figura 3 - Influência das lâminas de água em relação ao número de folhas para as mudas de amendoim-bravo (Pterogyne nitens Tul.).

As regressões também se ajustaram bem para esse parâmetro isolado com confiança de $98,4 \%$ para L1, 98,0\% para L2 e 98,8\% para L3. Segundo Lambers e Oliveira (2019), diversas espécies tem desenvolvido mecanismos para se adaptar a baixa quantidade de água no substrato, dentre eles o fechamento estomático, redução da taxa de fotossíntese e transpiração, redução da condutância sugerindo que a espécie de amendoim-bravo não necessita de irrigações por períodos prolongados (Silva et al., 2002).

Vários parâmetros vêm sendo estabelecidos a fim de determinar a qualidade de mudas em viveiro (Rudek et al., 2013). A importância da área foliar é amplamente conhecida por ser um indicativo de produtividade, fitomassa e da vida econômica do cultivo (Figueiredo et al., 2010). Pode-se mensurar esse parâmetro através dos métodos de contagem das folhas, dimensões foliares, método dos quadrados, dimensões lineares, com suas respectivas áreas foliares, e também através do uso de uma câmera digital, não havendo necessidade de equipamentos caros, conhecimentos avançados de matemática e tampouco da destruição das mudas (Rudek et al., 2013).

\section{Peso de matéria seca}

Na Tabela 02 é apresentado as médias obtidas pela combinação das variáveis substrato e lâmina de água pela qual observa-se que não há diferença estatística para matéria seca da parte aérea nem para matéria seca da raiz. Contudo a somatória das médias mostra uma biomassa total maior nos substratos S1 e S2 na L1.

Para a massa seca da parte aérea e do sistema radicular, foram observados maiores médias em S2 $(75 \%$ LEC + $25 \%$ SC) e S1 (100\% LEC) quando 
isoladas as lâminas de água.

Tabela 2 - Matéria seca para as mudas de amendoim-bravo (Pterogyne nitens Tul.), sob diferentes substratos (S) e lâminas de água (L).

\begin{tabular}{|c|c|c|c|c|c|c|c|c|c|}
\hline & & $\begin{array}{c}\text { MSPA } \\
\text { (g) }\end{array}$ & & & $\begin{array}{c}\text { MSR } \\
\text { (g) }\end{array}$ & & & $\begin{array}{c}\text { MST } \\
\text { (g) }\end{array}$ & \\
\hline & L1 & $\mathbf{L 2}$ & $\mathbf{L 3}$ & L1 & L2 & $\mathbf{L 3}$ & L1 & $\mathrm{L} 2$ & $\mathbf{L 3}$ \\
\hline S1 & $0,77 \pm 0,40$ & $0,78 \pm 0,28$ & $0,57 \pm 0,31$ & $1,23 \pm 0,66$ & $0,91 \pm 0,52$ & $0,90 \pm 0,40$ & $2,00 \mathrm{a}$ & $1,68 \mathrm{a}$ & $1,43 \mathrm{a}$ \\
\hline $\mathbf{S 2}$ & $0,79 \pm 0,18$ & $0,61 \pm 0,25$ & $0,73 \pm 0,27$ & $1,32 \pm 0,60$ & $0,67 \pm 0,27$ & $1,29 \pm 0,46$ & $2,11 \mathrm{a}$ & $1,28 \mathrm{a}$ & $2,02 \mathrm{a}$ \\
\hline S3 & $0,65 \pm 0,30$ & $0,63 \pm 0,29$ & $0,62 \pm 0,19$ & $0,91 \pm 0,31$ & $0,77 \pm 0,29$ & $0,92 \pm 0,41$ & $1,56 \mathrm{a}$ & $1,40 \mathrm{a}$ & $1,54 \mathrm{a}$ \\
\hline S4 & $0,42 \pm 0,14$ & $0,39 \pm 0,14$ & $0,39 \pm 0,09$ & $0,61 \pm 0,26$ & $0,60 \pm 0,24$ & $0,57 \pm 0,27$ & $1,03 a$ & $0,99 \mathrm{a}$ & $0,96 \mathrm{a}$ \\
\hline S5 & $0,26 \pm 0,10$ & $0,33 \pm 0,11$ & $0,33 \pm 0,13$ & $0,43 \pm 0,18$ & $0,36 \pm 0,15$ & $0,51 \pm 0,16$ & $0,69 \mathrm{a}$ & $0,69 \mathrm{a}$ & $0,84 \mathrm{a}$ \\
\hline
\end{tabular}

Legenda: Matéria seca da parte aérea (MSPA), matéria seca da raiz (MSR) e massa seca total (MST = MSPA+MSR). Letras iguais na horizontal significam que não há diferença estatística. L1: 6 minutos de irrigação (10 mm); L2: 9 minutos de irrigação (16 mm), L3: 12 minutos de irrigação $(21 \mathrm{~mm})$.

Em relação ao peso de MSPA, em L1 os tratamentos formulados com maiores concentrações de LEC, sendo eles S1 (100\% LEC), $\mathrm{S} 2(75 \% \mathrm{LEC}+25 \% \mathrm{SC})$ e S3 (50\% LEC + 50\% $\mathrm{SC)}$ apresentaram maiores médias de biomassa quando comparados com o controle (100\% SC), resultados semelhantes aos encontrados por Faria et al. (2013) em mudas de fedegoso gigante (Senna alata L.). Os autores também verificaram que na composição dos substratos para a produção de mudas, a adição do LEC resultou em maiores valores para altura da parte aérea, diâmetro do coleto e massa seca radicular.

A MSPA, segundo Gomes e Paiva (2006), é um indicador de rusticidade da muda, sendo que maiores valores representam mudas mais resistentes, o que prepara a muda para as adversidades que pode encontrar no campo.

Em L1, os tratamentos que apresentaram maiores pesos da matéria seca na raiz, foram os formulados com altas concentrações de LEC, sendo S2 (75\% LEC + 25 SC) e S1 (100\% LEC) ao ser comparado com o controle (100\% SC) (Figura 04).

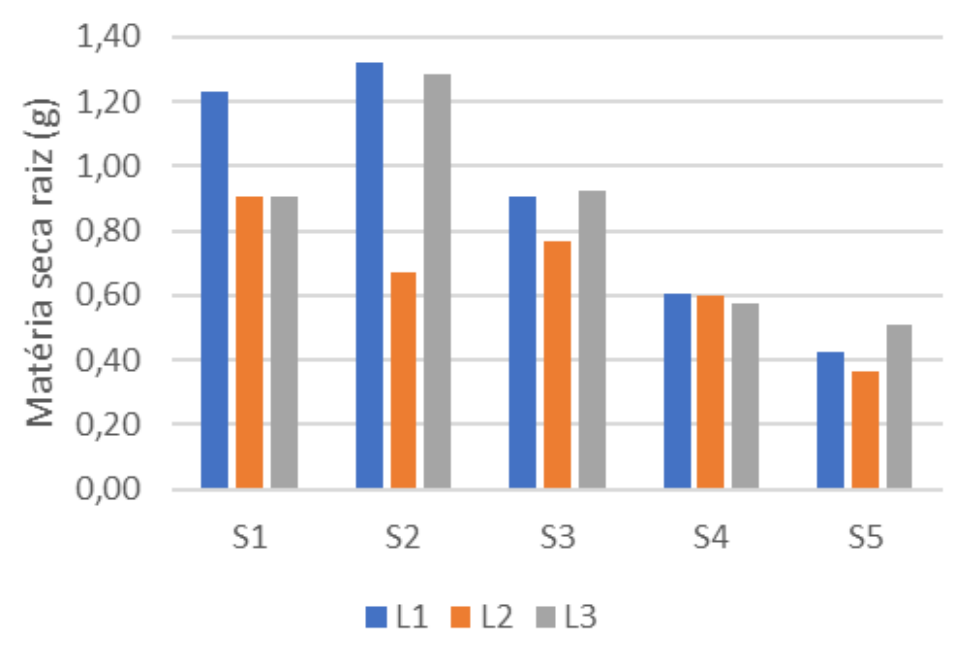

S1: 100\% LEC; S2: 75\% LEC + 25\% SC; S3: 50\% LEC + 50\% SC; S4: 25\% LEC + 75\% SC e S5: Controle 100\% SC. L1: 6 minutos de irrigação $(10 \mathrm{~mm})$; L2: 9 minutos de irrigação $(16 \mathrm{~mm}), \mathrm{L} 3: 12$ minutos de irrigação $(21 \mathrm{~mm})$.

Figura 4 - Influência dos substratos e lâminas de água em relação à matéria seca da raiz (MSR) das mudas de amendoim-bravo (Pterogyne nitens Tul.).

Para a MSR, os tratamentos com maior concentração de LEC: S1 (100\% LEC), S2 (75\% $\mathrm{LEC}+25 \mathrm{SC})$ e S3 (50\% LEC + 50\% SC), obtiveram os maiores valores médios, resultados também encontrados por Paiva et al. (2009) com cabreúva vermelha (Myroxylon peruiferum L.f.) e Caldeira et al. (2012) com teca (Tectona grandis L.f.). 
Para os valores de MST, os tratamentos formulados com concentrações de LEC, variando de $100 \%$ à $50 \%$, apresentaram resultados superiores em relação ao tratamento controle, formulado com $100 \%$ SC. Em relação as lâminas de água, todas obtiveram médias superiores em substratos formulados com altas concentrações de LEC, porém L1 se destacou, possuindo maiores concentrações de MST.

\section{Índice de Qualidade de Dickson}

O Índice de Qualidade de Dickson (IQD) é um indicador da qualidade da muda, considerando em seu cálculo a robustez (relação H/DC), o equilíbrio da distribuição de biomassa, fatores como biomassa aérea e da raiz, altura e DC (Dickson et al., 1960; Melo et al., 2018). Os resultados para o Índice de Qualidade de Dickson e a relação altura $(\mathrm{H})$ versus diâmetro do coleto (DC), encontram-se na Figura 05 .

Em relação à altura da parte aérea com H/DC, não houve diferença significativa entre os tratamentos, porém as maiores médias encontradas nos tratamentos S1/L2, S2/L1 e S3/L2 seguem o mesmo padrão dos demais dados analisados com valores médios maiores para as formulações de maior concentração de LEC (Figura 05).

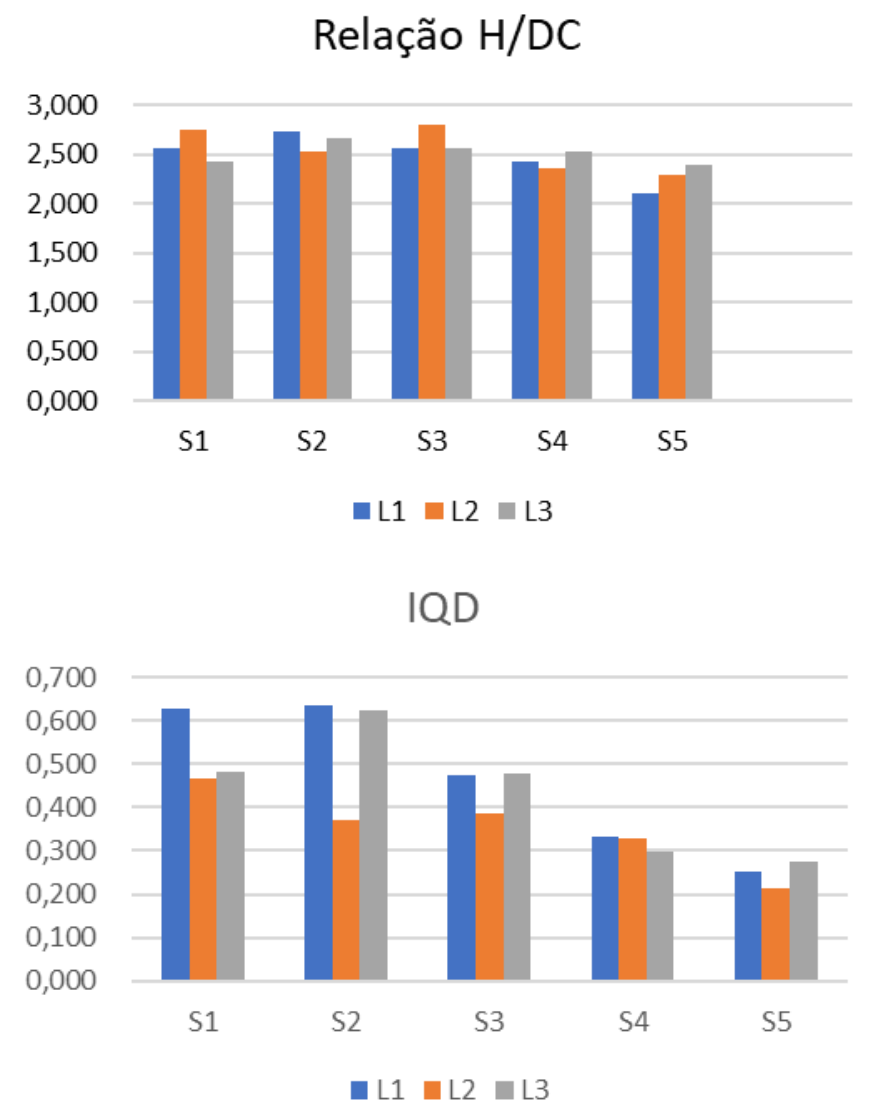

L1: 6 minutos de irrigação (10 mm); L2: 9 minutos de irrigação (16 mm), L3: 12 minutos de irrigação (21 mm).

Figura 5 - Valores do Índice de Qualidade de Dickson (IQD) e relação altura/diâmetro (H/DC) para mudas de amendoim-bravo (Pterogyne nitens Tul.).

De acordo com o IQD, os tratamentos S1 e S2 obtiveram resultados superiores em L1, apresentando uma diferença significativa em relação as outras lâminas de irrigação. É interessante constatar que as lâminas apresentam significância estatísticas apenas quando associados os parâmetros substrato e lâmina, demonstrando um sinergismo entre a irrigação e o substrato já que, isolatamente, a variação na irrigação não apresenta significância estatística para as medidas de número de folhas e altura das plantas.

O IQD é considerado importante para avaliação de mudas florestais, uma vez que avalia o acúmulo de reservas, assegura maior resistência e melhor fixação no solo, além de avaliar a capacidade de sobrevivência em campo (Sturion e Antunes, 2000). Para Caldeira et al. (2012), o IQD é uma característica que pode variar de acordo com a 
espécie, manejo, proporção dos substratos e idade fisiológica em que a muda foi avaliada.

Para o presente trabalho os índices mais indicados foram S1 (100\% LEC), S2 (75\% LEC + $25 \%$ SC) em L1 (6 minutos de irrigação). Por ser um índice muito variável, muitas vezes torna-se inviável comparar os resultados de IQD com estudo de diferentes autores

\section{CONCLUSÕES}

O LEC pode ser utilizado como substrato para a produção de mudas de amendoim-bravo, onde as proporções de $75 \%$ a $100 \%$ de LEC, na composição com SC, apresentaram os melhores valores em relação aos parâmetros morfológicos analisados.

$\mathrm{O}$ regime de irrigação, apresenta diferenças significativas apenas quando combinado com $\mathrm{o}$ substrato, mostrando sinergismo entre os tratamentos estudados, demonstrando que a L1 é suficiente para o desenvolvimento das mudas.

\section{AGRADECIMENTOS}

Ao Instituto Florestal de São Paulo pelo fornecimento das sementes. Ao Viveiro Associação de Recuperação Florestal e Ecológica ACIFLORA pelas facilidades concedidas no estabelecimento e monitoramento do experimento, bem como os revisores que contribuíram com suas importante sugestões para a versão final deste manuscrito.

\section{REFERÊNCIAS BIBLIOGRÁFICAS}

Alvares CA, Stape JL, Sentelhas PC, Gonçalves JL de M, Sparovek G. Köppen's climate classification map for Brazil. Meteorologische Zeitschrift, v.22, n.6, p.711-728, 2013. https://doi.org/10.1127/0941-2948/2013/0507

Bezerra FB, Oliveira MACL, Perez DV, Andrade AG, Meneguelli NA. Lodo de esgoto em revegetação de área degradada. Pesquisa Agropecuária Brasileira, v.41, n.3, p.469476, 2006. https://doi.org/10.1590/S0100204X2006000300014

Biruel RP, Borba Filho AB, Araújo ECE, Fraccaro FO, Perez SCJGA. Efeitos do condicionamento seguido ou não de secagem em sementes de Pterogyne nitens Tul. sob estresse. Ciência Florestal, v.17, n.2, p.119-128, 2007.

https://doi.org/10.5902/198050981943

Bomfim AA, Novaes AB, São José AR, Grisi FA. Avaliação morfológica de mudas de madeira-nova (Pterogyne nitens Tull.) produzidas em tubetes e sacos plásticos e de seu desempenho no campo. Floresta, v.39, n.1, p.33-40, 2009. http://dx.doi.org/10.5380/rf.v39i1.13723

Caldeira MVW, Peroni L, Gomes DR, Delarmelina WM, Trazzi PA. Diferentes proporções de biossólido na composição de substratos para produção de mudas de timbó
(Atleia glazioveana Baill). Scientia Forestalis, v.40, n.93, p.015-022, 2012.

Caldeira MVW, Delarmina WM, Peroni L, Gonçalves EO, Silva AG. Lodo de esgoto e vermiculita na produção de mudas de eucalipto. Pesquisa Agropecuária Tropical, Goiânia, v.43, n.2, p.155-163, 2013. https://doi.org/10.1590/S1983-40632013000200002

Cieslik BM, Namiesnik J, Konieczka P. Review of sewage sludge managements: standards, regulations and analytical methods. Journal of Cleaner Production, v.90, p.1-15, 2015. https://doi.org/10.1016/j.jclepro.2014.11.031

Dickson A, Leaf AL, Hosner JF. Quality appraisal of white spruce and white pine seedling stock in nurseries. Forest Chronicle, v.36, n.1, p.10-13, 1960. https://doi.org/10.5558/tfc36010-1

Faria JCT, Caldeira MVW, Delarmina WM, Lacerda LC, Gonçalves EO. Substratos à base de lodo de esgoto na produção de mudas de Senna alata. Comunicata Scientiae, v.4, n.4, p.342-351, 2013. https://doi.org/10.14295/cs.v4i4.242

Faustino R, Kato MT, Florêncio L, Gavazza S. Lodo de esgoto como substrato para produção de mudas de Senna siamea Lam. Revista Brasileira de Engenharia Agrícola e Ambiental, v.9, p.278-282, 2005.

Gomes JM, Paiva HN. Viveiros florestais: propagação sexuada. 3. Ed., Viçosa: UFV, p.116, 2004.

Lambers H, Oliveira RS. Plant Physiological Ecology. New York, Springer-Verlag. 3rd ed. 2019, XXVII, 736 p.

Lanzeti NGAA, Chimini AC, Neto MS, Paz MF, Siqueira MVBM. Lodo de esgoto compostado e diferentes lâminas de irrigações no desenvolvimento de Acacia polyphylla. Journal of Biotechnology and Biodiversity. v.9, n.2, p.201211, 2021.

https://doi.org/10.20873/jbb.uft.cemaf.v9n2.lanzeti

Leles PSS, Lisboa AC, Neto SNO, Grugiki MA, Ferreira MA. Qualidade de mudas de quatro espécies florestais produzidas em diferentes tubetes. Floresta e Ambiente, v.13, n.1, p.69-78, 2006.

Lobo TF, Oliveira FC, Morgado TB, Siqueira MVBM. Diferentes misturas de substrato com lodo de esgoto compostado enriquecido e substrato comercial em quaresmeira. Fronteiras: Journal of Social, Technological and Environmental Science, v.7, n.1, p.326-340, 2018. https://doi.org/10.21664/2238-8869.2018v7i1.p326-340

Lorenzi H. Árvores brasileiras: manual de identificação e cultivo de plantas arbóreas do Brasil. Nova Odessa: Instituto Plantarum, 2002.

Melo LA, Abreu AHM, Santos LPS, Oliveira RR, Silva DT. Qualidade e crescimento inicial de mudas de Mimosa caesalpiniifolia Benth. produzidas em diferentes volumes de recipientes. Ciência Florestal, v.28, n.1, p.47-55, 2018. https://doi.org/10.5902/1980509831574

Morais WWC, Susin F, Vivian MA, Araújo MM. Influência da irrigação no crescimento de mudas de Schinus terebinthifolius. Pesquisa Florestal Brasileira, v.32, n.69, p.2328, 2012. https://doi.org/10.4336/2012.pfb.32.69.23

Morgado BT, Olher I, Timo TPC, Rissi MN, Lobo TF, Siqueira MVBM. Growth of Cecropia hololeuca in water 
blades and substrates formulated with sewage sludge. Journal of Agricultural and Environmental Science/Revista de Ciências Agrárias, v.63, p.01-10, 2020.

http://dx.doi.org/10.22491/rca.2020.3174

Nobrega, M. A. S.; Pontes, M. S.; Santiago, E. F. Incorporação do lodo de esgoto na composição de substrato para produção de mudas nativas. Acta Biomedica Brasiliensia, v.8, n.1, p.43-55, 2017. https://doi.org/10.18571/acbm.121

Nogueira TAR, Melob WJ, Oliveira LR, Fonseca IM, Melo GMP, Marcussi AS, Marques MA. Nickel in soil and maize plants grown on an oxisol treated over a long time with sewage sludge. Abingdon, Chemical Special e Bioavailability, v.21, n.3, p.165-173, 2009. https://doi.org/10.3184/095422909X12470543670605

Olher I, Siqueira MVBM, Costa GHG, Lobo TF. Utilização de lodo de esgoto compostado e água residuária tratada para produção de pau viola. Journal of Biotechnology and Biodiversity, v.8, n.4, 304-310, 2020.

https://doi.org/10.20873/jbb.uft.cemaf.v8n4.olher

Oliveira LS, Araújo FM, Costa GHG, Lobo TF, Siqueira MVBM. Influência do lodo de esgoto compostado e de diferentes lâminas de água em mudas de mirindiba (Lafoensia glyptocarpa, Koehne). Pesquisa Florestal Brasileira, v.40, e201901948, p.1-6, 2020 https://dx.doi.org/10.4336/2020.pfb.40e201901948

Oliveira LR, Lima SF, Lima APL. Crescimento de mudas de cedro rosa em diferentes substratos. Pesquisa Florestal Brasileira, v.34, n.79, p.187-195, 2014 https://doi.org/10.4336/2014.pfb.34.79.605

Paiva AV, Poggiani F, Gonçalves JLM, Ferraz AV. Crescimento de mudas de espécies arbóreas nativas, adubadas com diferentes doses de lodo de esgoto seco e com fertilização mineral. Scientia Forestalis, v.37, n.84, p.499-511, 2009.

Pedrosa MVB, Lima WL, Amaral AA, Carvalho AHO. Biossólido de lodo de esgoto na agricultura: desafios e perspectivas. Revista Agrogeoambiental, v.9, n.4, p.125-142, 2017. http://dx.doi.org/10.18406/2316-1817v9n42017999

Rebouças JRL, Silva DN, Silva N, Silva MIG, Rajgheyi HANS, Neto ONS. Crescimento do feijão-caupi irrigado com água residuária de esgoto doméstico tratado. Revista Caatinga, v.23, n.1, 2010.

Rossa UB, Angelo AC, Westphalen DJ, Oliveira FEM, Silva FF, Araujo JC. Fertilizante de liberação lenda no desenvolvimento de mudas de Anadenathera peregrina (angico-vermelho) e Schinus terebinthifolius (aroeira-vermelha). Ciência Florestal, v.25, n.4, p.841-852, 2015.

https://doi.org/10.5902/1980509820582

Rudek A, Garcia FAO, Peres FSB. Avaliação da qualidade de mudas de eucalipto pela mensuração da área foliar com o uso de imagens digitais. Enciclopédia Biosfera, v.9, n.17, p.3775-3787, 2013.

Santana LO, Araújo FM, Souza FLP, Leme MC, Timo TPC, Lobo TF, Siqueira MVBM Desenvolvimento de mudas de dedaleiro (Lafoensia pacari. A.St.-Hil.) mediante diferentes substratos e lâminas de irrigação. Revista do Instituto Florestal, v.31, n.2, p.147-156, 2019.

Santos FEV, Kunz SH, Caldeira MVW, Azevedo CHS, Rangel OJP. Características químicas de substratos formulados com lodo de esgoto para produção de mudas florestais. Revista Brasileira de Engenharia Agrícola Ambiental, v.18, n.9, p.971-979, 2014. https://doi.org/10.1590/18071929/agriambi.v18n09p971-979

Santos MJC, Nascimento AVS, Mauro RA. Germinação do amendoim bravo (Pterogyne nitens Tul) para utilização na recuperação de áreas degradadas. Revista Brasileira de Ciências Agrárias, v.3, n.1, p.31-34, 2008. https://doi.org/10.5039/agraria.v3i1a182.

Santos CB, Longhi SJ, Hoppe JM, Moscovich FA. Efeito do volume de tubetes e tipos de substratos na qualidade de mudas de Cryptomeria japonica (L. F.) D. Don. Ciência Florestal, v.10, n.2, p.1-15, 2000. https://doi.org/10.5902/19805098466

Scheer MB, Carneiro C, Bressan AO, Santos KG. Compostos de lodo de esgoto para a produção de mudas de Anadenanthera colubrina (Vell.) Brenan. Cerne, v.18, n.4, p.613621, 2012. https://doi.org/10.1590/S010477602012000400011

Silva SRS, Demuner AJ, Barbosa LCA, Casali VWD, Nascimento EA, Pinheiro AL. Efeito do estresse hídrico sobre características de crescimento e a produção de óleo essencial de Melaleuca alternifolia Cheel. Acta Scientiarum, v.24, n.5, p.1363- 1368, 2002. https://doi.org/10.4025/actasciagron.v24i0.2382

Siqueira DP, Barroso DG, Marciano CR. Lodo de esgoto: diretrizes e o seu uso como fertilizantes, condicionador de solo e substrato florestal. Vértices, v.19, n.3, p.171-186, 2017. https://doi.org/10.19180/18092667.v19n32017p171-186

Siqueira DP, Carvalho GCMW, Barroso DG, Marciano CR. Lodo de esgoto tratado na composição de substrato para produção de mudas de Lafoensia glyptocarpa. Floresta, v.48, n.2, p. 277-284, 2018. http://dx.doi.org/10.5380/rf.v48i2.55795

Sturion JÁ, Antunes JBM. Produção de mudas de espécies florestais. In: Galvão APM (ed) Reflorestamento de propriedades rurais para fins produzidas e ambientais. Colombo: Embrapa Florestais, 2000. p.125-150.

Trigueiro RM, Guerrini IA. Utilização de lodo de esgoto na produção de mudas de aroeira-pimenteira. Revista Árvore, v.38, n.4, p.657-665, 2014. https://doi.org/10.1590/S010067622014000400009

Vega FVA, Bovi MLA, Berton RS, Godoy Junior G, Cembranelli MAR. Aplicação de biossólido na implantação da cultura da pupunheira. Horticultura Brasileira, v.22, p.131-135, 2004.

Weiss G, Ludvig A, Živojinović I. Four decades of innovation research in forestry and the forest-based industries - A systematic literature review. Forest Policy and Economics, v.120, 2020. https://doi.org/10.1016/j.forpol.2020.102288 